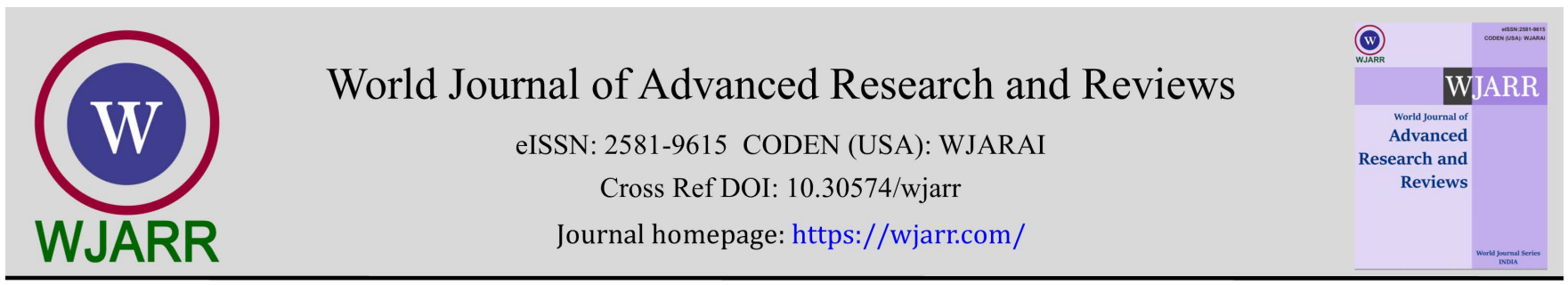

(REVIEW ARTICLE)

\title{
Correlation of fetal hemoglobin in different cancer patients and sickle cell anaemia: A review
}

\author{
Ayesha Shahid 1,* and Jan Peerzada Fawad ullah ${ }^{2}$ \\ ${ }^{1}$ Department of Pharmaceuticals sciences and Technology, Birla Institute of technology, Mesa, India. \\ 2 Department of Biochemistry, Hazara University, Mansehra, Pakistan.
}

World Journal of Advanced Research and Reviews, 2021, 12(01), 396-400

Publication history: Received on 25 August 2021; revised on 21 October 2021; accepted on 23 October 2021

Article DOI: https://doi.org/10.30574/wjarr.2021.12.1.0456

\begin{abstract}
Fetal hemoglobin is the main hemoglobin during gestation period. But this globin chain is replaced and is taken over by adult hemoglobin. Sometimes this switch from fetal to adult fails to occur leading to production of fetal hemoglobin as in case of sickle cell anemia. It is also observed that fetal hemoglobin expression is also seen under malignant condition. In malignancy the spleen, liver as well as gut acquire its ability to produce fetal hemoglobin. Certain HbF cells inducing factor such as stem cell growth factor and interleukin -3 also promote HbF erythropoiesis. HbF cells are indicated as a biomarker of tumour cells implicated in many carcinomas observed by immunohisto chemical investigations.
\end{abstract}

Keywords: Fetal hemoglobin; DNA methylation; Hematopoeisis; F cells

\section{Concept of Fetal Hemoglobin}

Fetal hemoglobin abbreviated as $\mathrm{HbF}$ is the vital hemoglobin found in human fetus. It starts during gestational period within first three months and constitute the major portion of hemoglobin in fetal growth. Approximately about $30-32$ week of gestational period the level of $\mathrm{HbF}$ starts lowering and the transition of switching to adult hemoglobin $\mathrm{HbA}$ takes place. At the time of birth hemoglobin is made up of $95 \%$ of fetal hemoglobin but eventually after child birth this get reduced to less than $1 \%$ and is preceded by adult hemoglobin constituting about $97 \%$ in adult.

\section{Production of fetal hemoglobin}

Fetal hemoglobin is a tetrameric molecule constituting of polypeptide subunits of two alpha and two gamma chain. The alpha subunit is present in chromosome number 16. The gene encoded by alpha subunit is HBA1 and HBA2 which produce identical proteins that differs only in regulatory region. The gamma subunit is present chromosome number 11. The gene encoded by gamma subunit is HBG1 and HBG2 which produce slightly different proteins. The initial switch is present in all mammals and involves a switch from haemoglobin subunits expressed exclusively in the transientlyproduced embryonic primitive wave of erythrocytes to the haemoglobin subunits produced in the earliest definitive wave of erythrocytes arising from the fetal liver [1]. The globin chain synthesis is transcribed by polymerase II .The messenger RNA present in the cytoplasm then translates into globin chain.The polyribosome on which the synthesis of globin chain occurs is proceeded to the very next stage owing to attachment with heme group .This further makes up the alpha and beta subunits.Switching of fetal to adult hemoglobin takes place due to silencing of gamma chain which plays a prominent role in fetal development. However from the fetal liver, fetal haemoglobin production is markedly increased [2]. During primate evolution, the genes encoding the fetal haemoglobin subunit were duplicated, such that there are two fetal globin genes in humans, HBG1 and HBG2, which differ by only a single amino acid. As the newborn

\footnotetext{
* Corresponding author: Ayesha Shahid

Department of Pharmaceuticals sciences and Technology, Birla Institute of technology, Mesa , India

Copyright (C) 2021 Author(s) retain the copyright of this article. This article is published under the terms of the Creative Commons Attribution Liscense 4.0.
} 
period approaches, the fetal switch begins to take place from HBG1 and HBG2 genes to the adult HBB gene [3] This transition of switch takes place normally and is completed during infancy. This lasts until approximately 6 months of age . It is investigated and reported that a non-anaemic individual continue to show a low level of HbF, which is found largely concentrated in a small percentage of erythrocytes being referred to as F cells [4]. In recent years the gene BCL11A has been identified that modulates the level of fetal hemoglobin being observed in chromosome 2p15[5].

\section{Mechanism involving $\mathrm{HbF}$ reactivation}

The synthesis of $\mathrm{HbF}$ and its expression occurs due to condition such as DNA methylation and due to malignancy condition taking place in spleen, liver and gut which often lead to regainment of fetal hemoglobin. There is evidence that $\mathrm{HbF}$ inducing growth factors are raised in cancer patients, including stem cell growth factor (CSF) and interleukin-3 in the serum of colorectal cancer patients [6]and a growth factor found in the bone marrow of patients with myelodysplastic syndrome [7].

\section{DNA Methylation}

The major etiology behind the change of Beta globin subunit to Gamma globin subunit in fetal development is due to major underlying changes that take place at molecular level. The major cause that form the basis of such mismatched globin chain is DNA methylation [8] This epigenetic phenomenon is useful for gene expression.In eukaryotes cytosine methylation is common.The promoter region of gamma globin chain has high concentration of cytosine nucleotide which is not present in promoter region of Beta globin. The substitution of methyl group at cytosine takes place at fifth position which yields 5-methyl cytosine with the help of enzyme DNA methyltransferase[9].This alteration takes place in pyrimidine ring at fifth position which is the place where DNA base thymine methyl group is positioned .It is the only position which differs Thymine from the RNA base Uracil that doesnot have methyl group .5-methylcytosine on spontaneous deamination converts to Thymine resulting in a mismatched ratio of Thymine :Guanine. There are many repair mechanism which comes into play so as to correct it into its original base pair Cytosine: Guanine. Further alternatively this leads to substitution of Adenine for Guanine which changes the base pair of Cytosine: Guanine pair into Thymine :Adenine pair .Change in such base pair give rise to point mutation. Gamma globin gene are found to be unmethylated in fetal liver tissue but methylated in adult bone marrow.

DNA hypomethylation is regarded as the major epigenetic process promoting neoplasia in tumour cells [10]. This often contributes to carcinogenesis and simultaneously leads to activation of gamma globin chain which ultimately express $\mathrm{HbF}$ gene in erythrocyte precursors in tumour tissue or in tissues where haematopoiesis take place.

\section{Malignant Condition}

Haematopoiesis refers to the formation of blood. In humans the process of haematopoiesis occurs via two developmental pathways i.e. primitive (embryonic) hematopoiesis occurs in blood islands in yolk sac [1] and generate enucleated RBCs that express embryonic Hbs while another is definitive hematopoiesis that takes place in fetal liver and gives rise to enucleated RBCs that express fetal hemoglobin [12]. Definitive (fetal/adult) hematopoiesis finally shifts from the fetal liver to the bone marrow where enucleated RBCs produce adult hemoglobin.

The concept of extramedullary haematopoiesis [13] i.e. formation of blood components outside of the medulla region of bone, comes into play. During certain pathological conditions as well as in malignancy the hematopoiesis doesn't work efficiently in the bone marrow and so ultimately the hematopoetic stem cells move to other tissue so as to continue the blood formation. Research and investigatory work reveal that there are some extramedullary organs like spleen and liver [14] which under pathological malignant condition regain the ability of fetal haematopoetic trait thus producing Hbf cells. In the same way the gut [15] can also regain the production of fetal hemoglobin which has hematopoetic progenitors produced during its 10-13 days of gestation period.

\section{HbF cells inducing factors}

There are stem cell growth factors [16] particularly cytokines that, stimulates proliferation of hematopoietic progenitor cell and enhance proliferative response to erythropoietin. The process of erythropoiesis is carried out through a pathway involving cytokine receptor that major role in differentiation and maturation of erythroid. The main cytokine receptor in the process of erythropoiesis is erythropoietin, which helps in survival as well as proliferation of late erythroid progenitors. The stem cell growth factors has the ability to activate fetal hemoglobin synthesis via a receptor called c-kit, belonging to tyrosine kinase family is expressed on CD34+hematopoietic progenitors [17]. These inducing 
factors stimulate and helps in survival and expansion of $\mathrm{f}$ cells. Subsequent studies and evidence also have shown that there is transforming growth factor Beta that has also caused an in increase in fetal hemoglobin synthesis along with erythropoiesis and stem cell growth factor.

Another growth factor is Interleukin 3, located on chromosome number 5. It is produced by stroma cells, monocytes,macrophages and activated $\mathrm{T}$ cells. The function of interleukin 3 is to stimulate the differentiation of multipotent hematopoietic stem cells into myeloid progenitor cells. Its high affinity receptor for human is constituted by alpha and beta subunit.

\section{Different types of $F$ cells found in cancers}

There are various kinds of blood cells found within fetal haemoglobin. The blood cells consists of erythrocytes which are mature and nucleated erythroblast.These are often observed in mitotic stage and in binucleated condition. The blood cells of fetal hemoglobin are seen and identified as in free state in tumour cells or in blood vessels. The various blood cells found include [18].

- FMLC, fetal haemoglobin-myeloid cells,

- FNBS, fetal haemoglobin normoblasts,

- FRBC, fetal haemoglobin-red blood cells;

Sources of F cells were identified and it was observed that there are two sources of Fcells [19]. The whole blood HbF is found to be enhanced in case of production in bone marrow while the elevated plasma $\mathrm{HbF}$ is found if the production takes place in tumour tissue. whole blood $\mathrm{HbF}$ owe its origin in circulating RBC and is recognized as inducible protein in many of the neoplasm.

\section{F cells found in different cancers}

A study was conducted to see the distribution of F cells in various types of cancers using immunohistochemical study [20].Many of them exhibited increased level of Hbf cells.

\subsection{Colorectal tumour}

HbF were found along with FRBC in some small blood vessels and capillaries.Some FNBS were also found among FRBC.A inverse correlation also existed between colorectal tumour as well as lymph nodes with respect to $\mathrm{HbF}$ cells. Some cases showed presence of $\mathrm{F}$ cells in tumour while no $\mathrm{F}$ cells in lymph nodes and vice versa.

\subsection{Nephroblastoma}

Fetal hemoglobin was observed in cases involving tumours consisting of nephroblastoma, which has epithelial cells forming tubular elements, blastoma cells and mesenchyme. Certain amount of F cells were found in between tumour cells and blood vessel that were proliferating.

\subsection{Retinoblastoma}

F cells were observed in many regenerating blood vessels and clusters of cells.

\subsection{Rhabdomyosarcoma}

F cells were found freely in tumour cells. In some cases F cells were found in clusters of bone marrow.

\subsection{Brain tumours}

F cells were not observed in majority of cases while FRBC were seen in tumour tissue of metastatic carcinoma.

\subsection{Medulloblastoma}

Many of the cases of cerebellum tumour showed presence F cells.

\subsection{Lung cancer}

The carcinoma of lung showed less concentration of FRBC in blood vessels. Here also inverse correlation of F cells were seen in tumour cells and lymph nodes. 


\subsection{Neuroblastoma}

Cases of Neuroblastoma showed presence of F cells. The cases also showed some fetal erythroblast being infiltrated in tumour cells as well as blood vessels.

\subsection{Breast cancer}

Cases with breast cancer showed no F cells in tumour cells while the lymph nodes revealed some amount of fetal RBC and fetal hemoglobin myeloid cells infiltrated in ductal carcinoma

\subsection{Myelodysplastic syndromes}

Cases with myelodysplastic syndrome showed fetal RBC, along with fetal hemoglobin normoblast, in the bone marrow.

\subsection{Trophoblastic disease}

In majority of trophoblastic disease cases, mature form of fetal hemoglobin myeloid cells and fetal hemoglobin normoblast were observed.

\section{Conclusion}

Fetal hemoglobin is a characteristic condition of sickle cell patients arising due to condition of DNA methylation. But this fetal hemoglobin blood cells are found and investigated under tumour condition indicating its presence in malignant condition as well. These F cells inside tumours are found to be of embryonic characteristics. The gene that express $\mathrm{HbF}$ accounts for BCL11A in humans. It express the human ontogeny of expressing HbF such as those of fetal and embryonic cell. Apart from DNA methylation there ae some other factors that leads to reactivation of $\mathrm{F}$ cells in adults, it can be malignant condition or Hbf inducing factor or even oncofetal gene. DNA hypomethylating agents is sometimes also regarded as a factor for initiating neoplasia among tumour cells along with reactivation of HbF gene expression occurring in erythrocytes precursors and also within the tumour tissues or in haematopoietic tissues. On evaluating observation of various tumour cells the result obtained showed that fetal hemoglobin are found in high concentration which indicates that it occurs as an inducible antigen rather than oncofetal antigen. A considerable level of fetal RBC is also expected to provide good and improved supply of oxygen in case of certain tumour. It is also concluded that the $\mathrm{F}$ cells in many cancers also result due to reactivation of oncofetal genetics that have occurred due to underlying microenvironment created. Identification of various hematological switching is still under research. Beside in future many hemoglobin blood cells are still on verge of ongoing studies to establish the correlation.

\section{Compliance with ethical standards}

\section{Acknowledgments}

The completion of this review article could not have been possible without the support of my co-author whose insightful knowledge and data analysis made this article a success.

\section{Disclosure of conflict of interest}

I and my co-author have no conflict of interest associated with this publication and there has been no significant financial support for this work that could have influenced its outcome. As the main author, I confirm that the manuscript has been read and approved for submission by my co-author.

\section{References}

[1] McGrath K, Palis J. Ontogeny of erythropoiesis in the mammalian embryo. Current Topics in Developmental Biology. 2008; 82: 1-22.

[2] Peschle C, Mavilio F, Care A, Migliaccio G, Migliaccio AR, Salvo G, Samoggia P, Petti S, Guerriero R, Marinucci M, Lazzaro D, Russo G, Mastroberardino G. Haemoglobin switching in human embryos: asynchrony of zeta-alpha and epsilon gamma-globin switches in primitive and definite erythropoietic lineage. Nature. 1985; 313: 235-238.

[3] Stamatoyannopoulos G. Control of globin gene expression during development and erythroid differentiation. Experimental Hematology. 2005; 33: 259-271. 
[4] Boyer SH, Belding TK, Margolet L, Noyes AN. Fetal hemoglobin restriction to a few erythrocytes (F cells) in normal human adults. Science. 1975; 188: 361-363.

[5] Sankaran VG, Xu J, Orkin SH. Advances in the understanding of haemoglobin switching. Br J Haematol. 2010; 149(2): 181-194.

[6] Mroczko B, Szmitkowski M, Okulczyk B. Hematopoietic growth factors in colorectal cancer patients. Clin Chem Lab Med. 2003; 41: 646-651.

[7] Choi JW, Kim Y, Fujino M, Ito M. F blast production correlates strongly with upregulation of inducible nitric oxide synthase in myelodysplastic syndromes. Ann Hematol. 2002; 81: 548 - 550.

[8] Ehrlich M. The controversial denouement of vertebrate DNA methylation research. Biochemistry (Mosc). 2005; 70: $568-575$.

[9] Okano M, Bell DW, Haber DA, Li E. DNA methyltransferases Dnmt3a and Dnmt3b are essential for de novo methylation and mammalian development. Cell. 1999; 99: 247-257.

[10] Atweh GF, Desimone J, Saunthararajha Y, Fathallah H, Weinberg RS, Nagel RL, Farby ME, Adams RJ. Hemoglobinopathies. Hematology (Am Soc Hematol Educ Program). 2003; 14 - 39

[11] Trimborn T, Gribnau J, Grosveld F, Fraser P. Mechanisms of developmental control of transcription in the murine alpha- and beta-globin loci. Genes and Development. 1999; 13: 112-124.

[12] Choi JW, KIM Y, et al. Hemoglobin F synthesis is not restricted to fetal erythropoietic organs during extramedullary hematopoiesis. Haematologica. 2002; 87: 323-5.

[13] Underwood JCE, Dangerfield VJM. Immunohistochemical identification of adult and fetal haematopoiesis in the spleen in lymphoma, leukaemia and myeloproliferative diseases. J Pathol. 1981; 134: 71-80.

[14] Gan OI, Semenova ML, et al. Development of day-8 colony forming unit-spleen hematopoietic progenitors during early murine embryogenesis: spatial.

[15] Wang W, Horner DN, Chen W, Zandstra PW, Audet J. Synergy between erythropoietin and stem cell factor during erythropoiesis can be quantitatively described without cosignaling effects. Biotechnol Bioengin. 2008; 99: 12611272.

[16] Gabbianelli M, Pelosi E, Bassano E, Labbaye C, Pedtti G, Rocca E, Tritarelli E, Miller BA, Valtieri M, Testa U, Peschle C. Granulocyte-macrophage colony-stimulating factor reactivates fetal hemoglobin synthesis in erythroblast clones from normal adults. Blood. 1989; 74: 2657-2669.

[17] Wolk M, Newland AC, De la Salle B, et al. Refinement of plasma fetal hemoglobin (HbF) measurements, as related to whole blood HbF, in cancer patients. J Tumor Marker Oncol. 1999; 14: 115-26.

[18] Wolk M, Newlands ES, Bar-Shira Maymon B, et al. Fetal hemoglobin detected serologically and immunohistochemically in cancer patients. J Tumor Marker Oncol. 2000; 15: 243-52.

[19] M Wolk, JE Martin, C Reinus. Development of fetal haemoglobin-blood cells (F cells) within colorectal tumour tissues Reinus. 9 February 2006. 